$\stackrel{N / N}{=}$

Global journals Inc.

है

\title{
Cytokine Storm: A Suicidal Immune Response to Novel Corona Virus
}

\author{
By Jignakumari J. Gohil \& Chiragkumar J. Gohil
}

Medical Officer

Abstract- SARS-CoV-2 virus is responsible for the COVID-19 disease in patients. Only $15-20 \%$ of COVID19 patients have developed severe pulmonary symptoms and illness, which are fatal to patients. Hyperimmune response to the SARS-CoV-2 virus by the host's immune system causes the release and over production of certain kinds of inflammatory mediators and cytokines. And it results in the cytokine storm. Cytokine storm produces the hyper inflammatory reaction, which deteriorates the cells and tissue. This type of immune response is host killing and suicidal response to the SARS-CoV-2 virus by the host. This suicidal response ultimately leads to lung damage, respiratory tract pneumonia, ARDS, multi-organ failure at a later stage and ultimately death. Hence, it needs to suppress the hyper-functioning of the immune system to inhibit the cytokine release and cytokine storm. Anti-inflammatory and immuno-modulatory drugs can be repurposed to manage the cytokine storm and hyper-immune response. Inhibition and management of the host's suicidal immune response and cytokine storm, could be life-saving and reduce the mortality rate in COVID-19 patients.

Keywords: sars-cov-2; covid-19; cytokine storm; hyperimmune response; ards; multi-organ failure; drugrepurposing.

GJMR-B Classification: NLMC Code: QW 640

Strictly as per the compliance and regulations of:

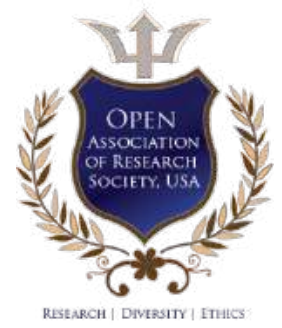

(C) 2021. Jignakumari J. Gohil \& Chiragkumar J. Gohil. This is a research/review paper, distributed under the terms of the Creative Commons Attribution-Noncommercial 3.0 Unported License http://creativecommons.org/licenses/by-nc/3.0/), permitting all noncommercial use, distribution, and reproduction in any medium, provided the original work is properly cited. 


\title{
Cytokine Storm: A Suicidal Immune Response to Novel Corona Virus
}

\author{
Jignakumari J. Gohil ${ }^{\alpha} \&$ Chiragkumar J. Gohil ${ }^{\sigma}$
}

\begin{abstract}
SARS-CoV-2 virus is responsible for the COVID-19 disease in patients. Only $15-20 \%$ of COVID-19 patients have developed severe pulmonary symptoms and illness, which are fatal to patients. Hyper-immune response to the SARS-CoV-2 virus by the host's immune system causes the release and over production of certain kinds of inflammatory mediators and cytokines. And it results in the cytokine storm. Cytokine storm produces the hyper inflammatory reaction, which deteriorates the cells and tissue. This type of immune response is host killing and suicidal response to the SARS-CoV-2 virus by the host. This suicidal response ultimately leads to lung damage, respiratory tract pneumonia, ARDS, multi-organ failure at a later stage and ultimately death. Hence, it needs to suppress the hyper-functioning of the immune system to inhibit the cytokine release and cytokine storm. Anti-inflammatory and immuno-modulatory drugs can be repurposed to manage the cytokine storm and hyper-immune response. Inhibition and management of the host's suicidal immune response and cytokine storm, could be life-saving and reduce the mortality rate in COVID-19 patients.
\end{abstract}

Keywords: sars-cov-2; covid-19; cytokine storm; hyperimmune response; ards; multi-organ failure; drugrepurposing.

\section{InTRODUCTION}

A novel corona virus named "Severe acute respiratory syndrome coronavirus-2" (SARS-CoV2) was identified in $2019-2020$ as a causative agent of several acute respiratory illness/infection [1]. This disease is caused by SARS-CoV-2, termed as "Coronavirus Disease 2019" (COVID-19). Covid-19 is a worldwide pandemic.

As theSARS-CoV-2 virus infects the cell, it copies itself very quickly. But the pathogenesis of the disease is still unclear. Because it produced a varying degree of clinical symptoms and illness in the various patients. In some of the patients, it only causes the asymptomatic forms and mild illness, while in some patients causes the fatal and life-threatening illness.

Among all COVID-19 patients, most of the patients are mildly affected and non-symptomatic. Some of the patients having mild symptoms like cough, fever, and muscle soreness. These types of patients, which are having mild symptoms at the early stage got severe

Author a: Medical Officer, COVID-19 Hospital, Ankleshwar 393001 , Gujarat, India.e-mail: jigna22196@gmail.com

Author o: Ph.D. Scholar, Discipline of Pharmacy, Gujarat Technological University, Ahmedabad 382424, Gujarat, India. to chronic illness and symptoms in the later stage of the disease or during the recovery process. The majority of critically ill patients did not develop severe clinically fatal complications in the early diseased stage.

Approximately 15-20 \% of the COVID-19 patients have developed pulmonary symptoms such as breathing difficulties and require hospitalization [2, 3]. In hospitalized patients, some of the patients show Acute Respiratory Distress Syndrome (ARDS) and multi-organ failure within 48-72 hours [4]. During the chronic illness stage, the ARDS and multiple organ failure occurs rapidly. These result in the death of the patients in a short time [5].

\section{a) Markers of COVID-19}

There is a reduction in the absolute lymphocyte count as well as a relative increase in neutrophillymphocyte ratio (NLR) in COVID-19 patients.

Elevation in C-reactive protein (CRP) and ferritin is also common.

Other markers of coagulation and organ damage such as D-dimer, lactate dehydrogenase $(\mathrm{LDH})$, creatinine and bilirubin are also observed to be elevated.

\section{b) Hyperimmune Response}

This is the question for the healthcare providers across the world, why only certain COVID-19 patients are having a chronic illness and severe symptoms. The answer is the hyper-immune response by the patient's immune system to the SARS-CoV-2 virus [5].

In many patients, the fatal damage has been occurred by the deranged or hyper immune response to the SARS-CoV-2 virus, rather than the virus itself. In many of the COVID-19 patients, their immune response has been as/more destructive as the virus that causes the disease.

It is seen the lung damage, severe respiratory damage and persistent high fevers in some critically ill COVID-19 patients. These are signs of hyper immune response and over functioning of the immune system.

Hyper-immune response induces the release of inflammatory proteins and mediators. Clinical studies show that a high level of immune system proteins has been found in the blood of the many seriously ill COVID19 patients.

Hence, it is clear that the release of these cytokines by the COVID-19 patient's immune system is 
responsible for the severe life-threatening clinical condition. Cytokine induces the aggravation of the disease and ultimately results in the cytokine storm.

Amount and levels of released cytokines in the cytokine storm; are injurious to host cells. This clinical condition is called disseminated intravascular coagulation (DIC) and it leads to a multi-organ system dysfunction. Cytokine storm is responsible for the ARDS and multiple organ failure in the stage of the chronic illness [7].

Along with the COVID-19, hyper-immune response and release of immune cytokine proteins have been also occurring in some clinical conditions like: In infections like flu, H1N1 influenza, during certain kinds of cancer treatments (chemotherapy), and in autoimmune diseases (juvenile arthritis).

Clinical data evidence that; the amount of the cytokines produced by cells in response to a SARSCoV-2 infection is about 50 times higher than the number of cytokines produced in response to West Nile virus or Zika virus infections.

Cytokine storm generally observed in the cytomegalovirus, Epstein-Barr virus-associated hemophagocytic lymphohistiocytosis, group A streptococcus, influenza virus, variola virus and in the SARS-CoV-2 virus [8].

\section{il. Cytokines and Cytokine Storm}

Cytokine was first time coined in the context of avian H5N1 influenza virus infection in 2005 [9]

The word cytokines composed of two words, "Cyto (cell)" and "Kines (small proteins)". Cytokines are an essential part of the inflammatory process. Cytokines are a diverse group of the protein which are being secreted by the immune cells. Cytokines are produced by certain types of immune cells like macrophages, dendritic cells, natural killer cells, T and B lymphocytes.

Cytokines have an autocrine, paracrine, and/or endocrine activity. And it can elicit a wide range ofresponses (through receptor binding) depending upon the types of cytokine and target cell [10].

The principal role of the cytokine is intercellular signaling and communication. The main functions of cytokines are control of cell proliferation and differentiation, regulation of angiogenesis and immune and inflammatory responses. Apart from these, it also performs various functions in the body.

\section{a) Cytokine Storm}

Cytokine storm has defined by hyperactive immune-response to SARS-CoV-2 virus by the host's immune system. It consists of the release of interferons, interleukins, tumor-necrosis factors, chemokines, and several other mediators [11].

These released soluble markers of inflammation are certain kinds of proteins of the immune system, called cytokines. These mediators are part of a wellconserved innate immune response, which is necessary for the efficient clearance of virus and infectious agents Generally, the anti-viral immune response requires the activation of the inflammatory pathways of the immune system. If this immune response remains uncontrolled, the hyper immune response can cause severe clinical manifestation [12]

\section{i. Mechanism}

When the immune system (macrophages and some other kinds of immune cells) of the host/patient detects the antigen of the SARS-CoV-2virus, they send the messages by releasing various proteins (cytokines). This immune response to the SARS-CoV-2 virus initiates the sequence of events results in the recruitment of leukocytes and plasma proteins (cytokines) to the site of infection [13].

These cytokines will produce and release the other immune cells to initiate an inflammatory response. This inflammatory immune response which is initiated by the cytokines helps the body to fight against the SARSCoV-2virus [14].

When the host's immune system hyper reacts to the SARS-CoV-2 virus, macrophages can also release other signaling molecules (catecholamines). These catecholamines trigger the release of more cytokines and intensify the inflammatory response. Systemic hyper-inflammation elevates the level of various cytokines such as TNF, IL-2, IL-7, IL-6, IP-10, IFN- $\gamma$, MIP1 $\alpha$, MIP1 $\beta$ and MCP1. Ultimately there is a large amount of the cytokines release in the COVID-19 patient. This flux of the cytokines is known as a "Cytokine storm".

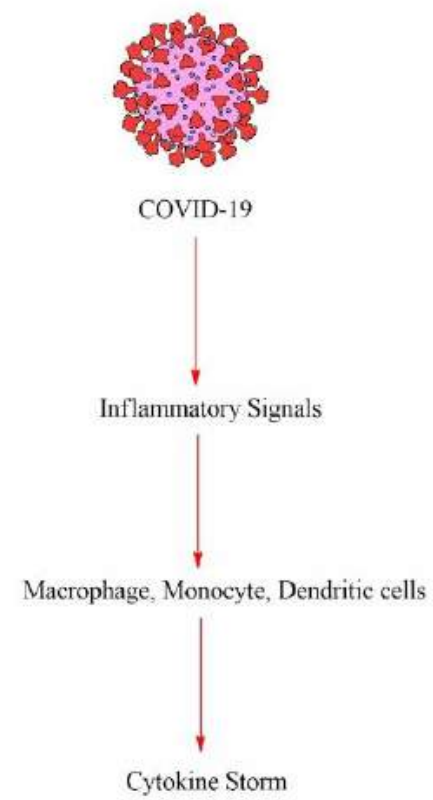

Figure 1: Hyperimmune response and Cytokine Storm ${ }^{[15]}$ 
These clinical conditions and responses are referred to as macrophage activation syndrome, cytokine release syndrome or simply cytokine storms.

$\|-1$, TNF-a, and IL-6 are the main proinflammatory cytokines in the immune response to the SARS-CoV-2 virus. In turn, an increase in the level of these three cytokines also induces the adhesion of the macrophages, neutrophils, and $T$ cells from the circulation into the site of infection. This cascade of mechanisms produces destructive effects on human tissue. And causes the destabilization of endothelial cell to cell interactions, damage of vascular barrier, capillary damage, diffuse alveolar damage. Cytokine storm induces lung injury [16]. And if the cytokine storm is not controlled and left untreated, then patients will have ARDS as a result of acute lung damage followed by multi-organ failure and death.

\section{iil. Role of Interleukins in Cytokine STORM}

Previously, it was thought that Interleukins are the cytokines that are produced by the leukocytes. But now, it is clear that interleukins are produced by a wide variety of cell types.

In contrast to the IFNs, the interleukins are a diverse family of immune system regulators. Their main role is in immune cell differentiation and activation. The prime role of the interleukins is in intercellular communication [17]. They also control the growth and differentiation of the leukocytes. As of the other cytokines, it elicits the wide range of the response [18].

In the interleukin family, IL-1 and IL-6 are proinflammatory cytokines. It mediates the host's immune response to SARS-CoV-2 virus infection through direct and indirect mechanisms [19]. Receptor signaling of IL1 is responsible for acute lung immunopathology in COVID-19patients [20].

\section{a) Crucial Role of IL-6}

Interleukin-6, a pro-inflammatory cytokine, is a key mediator in the acute inflammatory response and the purported cytokine storm. Increased level of IL-6 in the serum have been correlated with respiratory failure, ARDS and adverse clinical outcomes [21].

\section{iv. Cytokines Storm and Lung Damage}

Many studies have been carried out to find; whether drugs and treatment of COVID-19 provoke the cytokine storm or not.

Cytokine storm is the situation of the hyper immune response to the antigen, where the body starts attacking its own cells and tissues rather than fighting off the antigen (virus) [22].

Cells kill themselves (suicidal response); when they sense the antigen or virus enters the cells and begin to infect them. This type of self-killing response is a protective mechanism, so the virus does not spread to the other cells [23].

When the cells can't cope up with the antigen/virus they begin to send SOS signals through immune proteins and chemical messengers. These immune proteins and chemical messengers are a certain type of cytokines. The release of cytokines triggers cell death. A large number of virus-infected cells release the cytokines at the same time, so a larger number of tissues can die. In COVID-19 patients, this phenomenon is mostly seen in the lung tissues [24].

This hyper-inflammatory condition consists of the pulmonary infiltration and exudation, organ damage and dysfunction of coagulatory pathways, which leads to the worsening of oxygen saturation.

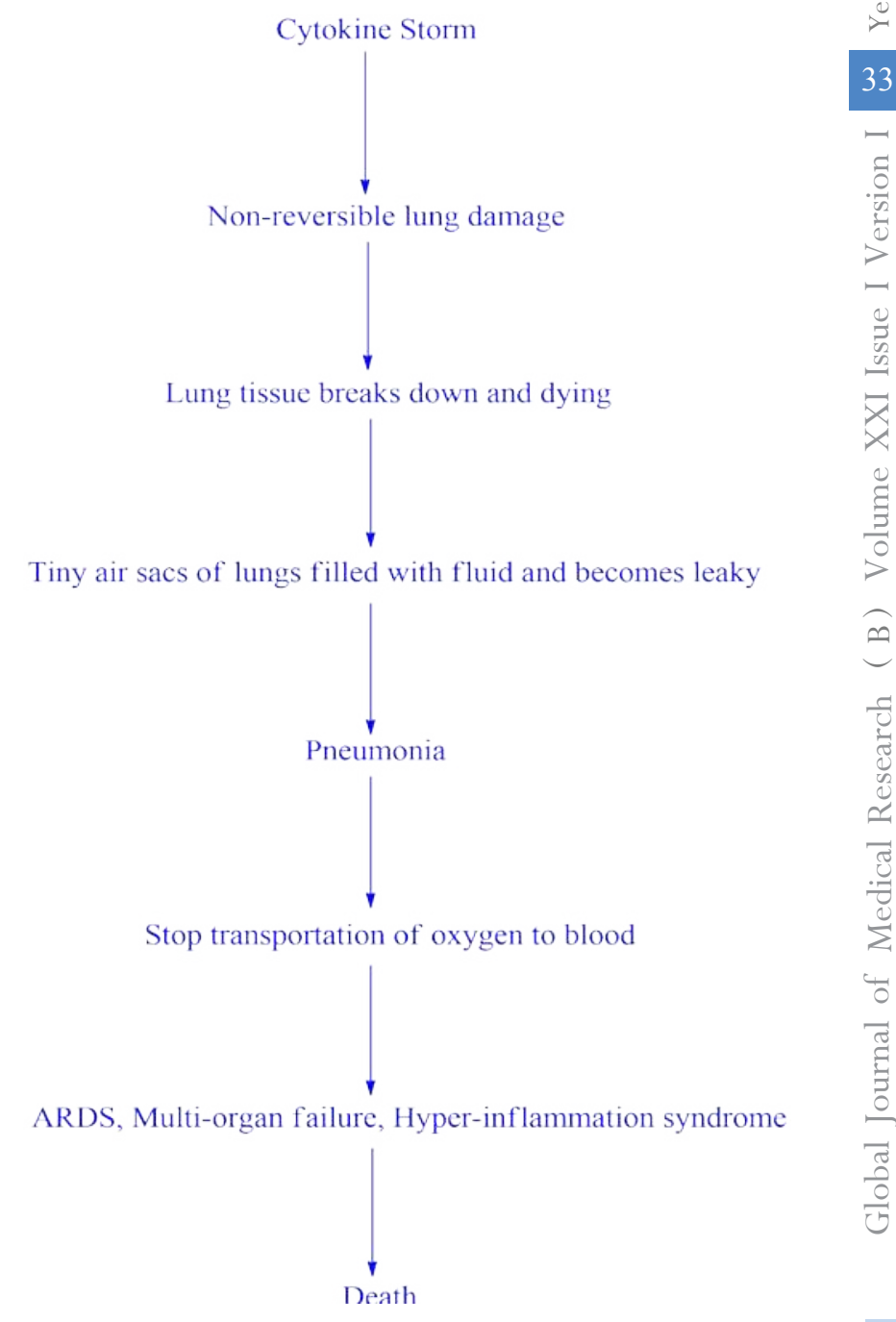

Figure 2: Lung damage by Cytokine storm ${ }^{[25]}$

Cytokines and chemical mediators eat away at the lung and the lung becomes greatly damaged, in a non-reversible manner. As the lung's tissue starts dying and breaks down, the lung's wall (tiny air sacs) is filled with the fluid and becomes leaky. So, this causes pneumonia and stops the transport and supply of 
oxygen to the blood and body. Hence, the body will be starving of oxygen and respiratory distress syndrome follows. Then other organs start to fail.

This clinical condition plays a central role in the death of a large number of COVID-19 patients.

\section{Treatment of Cytokine Storm}

Cytokine Storm (CS) is a lethal clinical condition, and it requires intensive care; since it is responsible for the high mortality rate in COVID-19 patients [26, 27].

As cytokine storm is a chronic inflammatory condition induced by the hyper immune response of the host, the inflammation must be treated and control to cope the condition.

Basically, anti-inflammatory drugs, a combination of immune-modulators, and some other drugs can be used. IL-6 inhibitors and high dose corticosteroids block pathways critical to host immune responses. Many monoclonal antibody drugs are being repurposed from treating patients with chronic inflammatory conditions [28, 29].

\section{a) Catecholamine inhibitors}

Before the cytokine storm, the circulating level of catecholamines increases rapidly in the COVID-19 patients. This catecholamine is responsible for the cycle of inflammation and cytokine storm. If we inhibit the release of the catecholamine, we can inhibit the cytokine storm. We can use and test the catecholamine inhibitor drugs to treat COVID-19 patients.

\section{b) Alpha-blockers}

Various studies on mice and other findings show that the alpha-blockers can be used to break the hyper-inflammatory chain reaction. So, we can prevent it from converting to cytokine storm. Generally, alphablocker drugs are used for the treatment of prostate disease and high blood pressure.

\section{i. Mechanism of action}

Cell signaling and communication have occurred through the cytokines and chemical mediators. Alpha-blocker drugs interfere with this cell signaling during the inflammatory immune response and inhibitit. Alpha-blockers break the chain of cytokine release and inhibit the cytokine storm.

Data indicate that, COVID-19 patients taking the regular and gradual dose of an alpha-blocker (prazosin) have no sign of the hyper inflammatory immune response or cytokine storm.

\section{c) Anti-rheumatic drugs}

Another approach to control the hyperinflammatory immune response is to use anti-rheumatic drugs.

In a study, COVID-19 patients are treated with I.V infusions of "Actemra (anti-rheumatic)". In these patients, actemra drug blocks the cytokine IL-6 receptor and decreases the circulating level of IL-6. So actemra successfully inhibits the cytokine storm in COVID-19 patients. These results encourage the researchers to test another anti-arthritis drug names "auranofin".

\section{d) Chloroquine and Hydroxy-chloroquine}

Chloroquine and hydroxychloroquine have a variety of biological activity from antimalarial, antiviral to anti-inflammatory. Because of the anti-inflammatory property, they are used in the therapy of autoimmune diseases.

Cytokine storm is a similar condition to the autoimmune disease. So these drugs can be effective in the management of the cytokine storm in the COVID-19 patients.

\section{i. Mechanism of action}

Chemically,

chloroquine; and hydroxychloroquine are weak bases in nature. So they used to accumulate in acidic organelles like lysosomes. And they increase lysosomal/endosomal $\mathrm{pH}$. So, chloroquine and hydroxychloroquine can decrease the production of various pro-inflammatory cytokines. Therefore, prevent the cytokine storm [30] and inhibit replication of the SARS-CoV-2 virus [31].

\section{e) Corticosteroids}

Corticosteroids can be used as an antiinflammatory drug in COVID-19 patients. Corticosteroids down-regulate the hyper-activity of the immune system and inhibit the release of the cytokine and cytokine storm.

Corticosteroids are most widely used in the treatment of many infectious diseases. But, the use of corticosteroids drugs in the treatment of COVID-19 can cause host immune suppression and delay of viral clearance.

\section{f) IL-6-IL-6R antagonists}

IL-6 is a potential target in the treatment of cytokine storm because IL-6 plays the central role in the cytokine release and hyper-inflammatory cytokine storm. Many studies have been carried out using the IL-6-IL-6R antagonist drugs to treat COVID-19 patients. Treatment with IL-6-IL-6R antagonists drugs shows that; drugs inhibit the cytokine chain reaction And decreases the level of the pro-inflammatory cytokine IL-6. In turn, a fall in the circulating level of the cytokines. Hence, inhibiting the cytokine storm. And shown a good patient prognosis.

\section{g) Monoclonal antibody}

Elevated levels of the IL-6 in the blood of COVID-19 patients; have been reported to be predictive of a lethal outcome in patients [32].

Therefore, it needs to be harnessed the increasing level of II-6 in the COVID-19 patient to prevent the cytokine storm and to save a patient's life. In this 
situation, a monoclonal antibody can be used to block the IL-6.

\section{i. Tocilizumab}

Tocilizumab is a recombinant humanized monoclonal antibody; and it is an IL-6 receptor antagonist.

Tocilizumab (specific monoclonal antibody) therapy has been used to treat the cytokine storm and critically ill patients with extensive lesions in bilateral lungs and a confirmed elevated level of IL-6.

Elaborate studies on 20 severe patients of COVID-19 indicate that the tocilizumab drug effectively blocks IL-6. And it leads to a reduction in fever and lung lesion opacity; and recovered the percentage of lymphocytes in peripheral blood [33].

On the other hand, there is a risk of opportunistic infections like tuberculosis, fungal, and other viral infections caused by anti-IL-6 monoclonal antibodies.

\section{ii. Itolizumab}

Itolizumab is an immune-modulator drug. Itolizumab is an anti-CD6 humanized IgG1 monoclonal antibody (mAb). It can potentially inhibit the cytokine storm and is found as a life-saving drug in COVID19patients.

It is an approved drug for the treatment of psoriasis in India [34, 35].

\section{- Mechanism of action}

It binds with T-effector cells through domain-1 of the CD6 receptor present on it. Domian-1 of T-effector cells is responsible for priming, activation, and differentiation of T-Cells [36].

Itolizumab's binding blocks the co-stimulation pathway and leads to inhibition of proliferation of T-cells. It consequently reduced the level of pro-inflammatory cytokines (IL17A, TNF- $\alpha$, IL-6, IFN- $\gamma$, and IL-2). And brings down the release of multiple cytokines and cell signaling transduction and inhibits the cytokine storm.

After the whelming result in a study on COVID19patients, it is under clinical trials to treat COVID-19.

\section{Conclusion}

The immune system of some COVID-19 patients gives a suicidal immune response to the SARSCoV-2 virus. Which excessively release inflammatory cytokines and proteins.

The high circulatory level of cytokines known as a cytokine storm; initiates the chronic inflammatory reaction in the body (mostly in the lung). Cytokine storm leads to physiological deterioration of the lung tissue and causes ARDS, multiple-organ dysfunction, and death.

Particular pro-inflammatory cytokine IL-6 is the marker of the cytokine storm. The blood level of the IL-6 is correlated with cytokine storm in the body. The normal level of the IL-6 in the blood is <7, an IL-6 level more than 7 indicating the cytokine storm.

So timely control of the cytokine storm in its early stage would be beneficial and can save the patient's life.

Corticosteroids, anti-rheumatoid drugs, and many other anti-inflammatory drugs have been repurposed to treat the cytokine storm and hyper inflammatory condition inCOVID-19 patients. The use of newer IL-6 inhibitors/antagonist drugs has also been proved effective in the treatment of the cytokine storm.

Target-specific monoclonal antibody drugs have been used to decrease the hyper-functioning of the patient's immune system. These humanized monoclonal antibody drug show impressive results in the management of severe clinical conditions in the patients.

Currently there are no approved drugs and therapies available for the treatment of COVID-19 patients. Several marketed drugs have been repurposed for the treatment of cytokine storm. And many immunemodulator biologics have been used off label for the management of the hyper/suicidal immune response in the COVID-19 patients.

All the results of these new monoclonal antibody drugs are preliminary. There are no randomized clinical trials of the newer monoclonal antibody drugs that have been published to date, so it requires clinical trials and confirmatory studies to evaluate its role in COVID-19 therapy.

\section{ACKNOWLEDGments}

Co-author thank Dr. Jignakumari J. Gohil for providing details of the cytokine storm in COVID-19 patients and repurposing of the drugs for its treatment.

\section{References Références Referencias}

1. Gohil CJ., Noolvi MN."Novel Corona Virus and Its Druggable Targets". Curr Opin Virus Infect Dis.2020; 1(3): 51-53.

2. Wang D., Hu B., $\mathrm{Hu}$ C., et al. "Clinical Characteristics of 138 Hospitalized Patients With 2019Novel Coronavirus-Infected Pneumonia in Wuhan, China". JAMA. 2020; 323: 1061-1069.

3. Sen DJ., Mandal SK., Biswas A., Dastider D., Mahanti B. "Corona is Culprit of Ridiculous Offensive Nonsense Air". World J Pharm Res. 2020; 9: $1-16$

4. Tay MZ., Poh CM., Renia L., Macary PA., Ng LFP. "The trinity of COVID-19: immunity, inflammation and intervention". Nat Rev Immunol. 2020; 1-12.

5. Qin C., Zhou L., Hu Z., Zhang S., Yang S., Tao Y.,Xie C., Ma K., Shang K., Wang tian DS. "Dysregulation of immune response in patients with Coronavirus 
2019 (COVID-19) in Wuhan, China". Clin Infect Dis. 2020; 71: 762-768.

6. Ye Q., Wang B., Mao J."The pathogenesis and treatment of the 'Cytokine Storm' in COVID-19".J Infect. 2020; 80: 607-613.

7. Huang KJ., Sul J., Theron M., Wu YC., Lai SK., Liu CC., Lei HY. "An interferon-gamma-related cytokine storm in SARS patients". J Med Virol. 2005;75: 185194.

8. Prajapat M., Sarma P., Shekhar N., Avti P., Sinha S., Kaur H., Kumar S., Bhattacharyya A., Kumar H., Bansal S., Medhi B."Drug targets for corona virus: A systematic review". Indian J Pharmacol. 2020; 52: 56-65.

9. Yuen KY., Wong SS."Human infection by avian influenza A H5N1". Hong Kong Med J. 2005; 11:189 -199 .

10. Coperchinia F., Chiovatoa L., Crocea L., Magria F., Rotondia M. "The cytokine storm in COVID-19: An overview of the involvement of the chemokine /chemokine-receptor system". Cytokine Growth Factor Rev. 2020; 53: 25-32.

11. Sinha P., Matthay MA., Calfee CS. "Is a "Cytokine Storm" Relevant to COVID-19?". JAMA Intern Med. 2020; E1-E3.

12. BracialeT., Hahn Y. "Immunity to viruses". Immunol Rev. 2013; 255: 5-12.

13. Thompson M., Kaminski J., Kurt-jones E., Fitzgerald K. "Pattern recognition receptors and the innate immune response to viral infection". Viruses.2011; 3:920-940.

14. Loganathan S., Athalye SN., Joshi SR."Itolizumab, an anti-CD6 monoclonal antibody, as apotential treatment for COVID-19 complications". Expert Opin Biol Ther. 2020.

15. Mehta Y., Dixit SB., Zirpe KG., Ansari AS. "Cytokine Storm in Novel Coronavirus Disease (COVID-19): Expert Management Considerations". Indian J Crit Care Med. 2020; 24: 429-434.

16. Prarthana MS., Kalagi S. "Middle East Respiratory Syndrome: An Overview". Int J Health Sci Res. 2015; 5: $422-429$.

17. Ragab D.,EldinHS., TaeimahM.,Khattab R.,Salem R. "The COVID-19 Cytokine Storm; WhatWe Know So Far".Front Immunol. 2020;11: 1-4.

18. TechNotes. COVID-19 and the Cytokine Storm: The Crucial Role of IL-6. Enzo life sciences, [cited 2020 Sep 21],https://www.enzolifesciences.com/sciencecenter/technotes/2020/april/covid-19-and-the-

cytokine-storm-the-crucial-role-of-il-6/

19. Dinarello CA. "Immunological and inflammatory functions of the interleukin-1 family". Annu Rev Immunol.2009; 27:519-550.

20. Schmitz N., Kurrer M., Bachmann MF., Kopf M."Interleukin-1 is responsible for acute lung immunopathology but increases survival of respiratory influenza virus infection". J Virol. 2005; 79:6441-6448.

21. Tisoncik JR., Korth MJ., Simmons CP., Farrar J., Martin TR., Katzea MG."Into the Eye of the Cytokine Storm". Microbiol. Mol Biol Rev. 2012; 76: 16-32.

22. Goodman B. Cytokine Storms May Be Fueling Some COVID Deaths. WebMD Health News, [cited 2020 Sep 22],https://www.webmd.com/lung/news/ 20200417/cytokine-storms-may-be-fueling-somecovid-deaths

23. Zhao M. "Cytokine storm and immunomodulatory therapy in COVID-19: Role of chloroquine and antiIL-6 monoclonal antibodies". Int J Antimicrob Agents. 2020; 55:1-3.

24. Science News. Preventing 'cytokine storm' may ease severe COVID-19 symptoms. Howard Hughes Medical Institute, Available online: https://www. sciencedaily.com/releases/2020/05/200521165706. $\mathrm{html}$ (accessed on 17 November 2020).

25. Jain A. "COVID-19 and lung pathology". Indian J Pathol Microbiol. 2020; 63: 171-172.

26. Ishikawa T. "Clinical preparedness for cytokine storm induced by the highly pathogenic H5N1 influenza virus". J Pharmacogenom Pharma coproteomics.2012; 3: 1000e131.

27. Kalaiyarasu S., Kumar M., Senthil kumar D., Bhatia S., Dash SK., Bhat S., et al. "Highly pathogenic avian influenza $\mathrm{H} 5 \mathrm{~N} 1$ virus induces cytokine dysregulation with suppressed maturation of chicken monocyte-derived dendritic cells". Microbiol Immunol. 2016; 60: 687-693.

28. Roschewski M., Lionakis MS., Sharman JP., et al. "Inhibition of Bruton tyrosine kinase in patients with severe COVID-19". Sci Immunol. 2020;5(48), https://doi.org/10.1126/sciimmunol.abd0110

29. Alijotas-reig J., Esteve-valverde E., Belizna C., Selva-o'callaghan A., Pardos-gea J., Quintana A., Mekinian A., Anunciacion-llunell A., Miro-mur F. "Immunomodulatory therapy for the management of severe COVID-19. Beyond the anti-viral therapy: A comprehensive review". Autoimmun Rev. 2020; 19, https://doi.org/10.1016/j.autrev.2020.102569

30. Schrezenmeier E., Dorner T."Mechanisms of action of hydroxychloroquine and chloroquine: implications for rheumatology". Nat Rev Rheumatol.2020; 16: 155-166.

31. Devaux CA., Rolain JM., Colson P., Raoult D. "New insights on the antiviral effects of chloroquine against coronavirus: what to expect for COVID19?".Int J Antimicrob Agents.2020; 55, https://doi. org /10.1016/j.ijantimicag.2020.105938

32. Ruan Q., Yang K., Wang W., Jiang L., Song J. "Clinical predictors of mortality due to COVID-19 based on an analysis of data of 150 patients from Wuhan, China". Intensive Care Med. 2020; 46:846848. 
33. Xu X., Han M., Li T., Sun W., Wang D., Fu B., Zhou Y., Zheng X., Yang Y., Li X., Zhang X., Pan A., Wei $\mathrm{H}$." Effective treatment of severe COVID-19 patients with tocilizumab". Proc Natl Acad Sci USA. 2020; 117: 10970-10975.

34. Anand A., Assudani D., Nair P., Krishnamurthy S.,Deodhar S., Arumugam M., lyer H., Melarkode R. "Safety, Efficacy and pharmacokinetics of T1h, a humanizedanti-CD6 monoclonal antibody, in moderate to severe chronic plaque psoriasisResults from a randomized phase II trial.(96.13)". J Immunol. 2010; 184, https://www.jimmunol.Org /content/184/1_Supplement/96.13

35. Krupashankar D., Dogra S., Kura M., et al. "Efficacy and safety of itolizumab, a novel antiCD6monoclonal antibody, in patients with moderate to severe chronic plaque psoriasis: results of a double-blind, randomized, placebo-controlled, phase-III study". J Am Acad Dermatol. 2014; 71:484-492.

36. Nair P., Melarkode R., Rajkumar D., Montero E. "CD6 synergistic co-stimulation promoting proinflammatory response is modulated without interfering with the activated leucocyte celladhesion molecule interaction". Clin Exp Immunol. 2010; 162: 116-130.

Note

SARS-CoV-2 virus and Novel Corona Virus, both the terms are similar.

Author Contributions

Dr. Jignakumari J. Gohil contributed to providing clinical data of COVID-19 patients.

Co-author, Chiragkumar J. Gohil contributed to writing, editing, and revising of the manuscript. 